\title{
Tracheoesophageal Voice Prosthesis Use and Maintenance in Laryngectomees
}

\author{
Itzhak Brook ${ }^{10}$ Joseph F Goodman² \\ ${ }^{1}$ Department of Pediatrics/Medicine, Georgetown University, \\ Washington, District of Columbia, United States \\ 2 Department of Otolaryngology, George Washington University \\ School of Medicine, Washington, District of Columbia, United States \\ Int Arch Otorhinolaryngol 2020;24(4):e535-e538.
}

\author{
Address for correspondence Itzhak Brook, MD, MSc, 4431 Albemarle \\ St NW, Washington, DC, 20016, United States \\ (e-mail: ib6@georgetown.edu).
}

\author{
Abstract \\ Keywords \\ - tracheoesophageal \\ voice prosthesis \\ - tracheoesophageal \\ prosthesis \\ maintenance \\ - laryngectomees \\ - sputum \\ - speech outcomes
}

Tracheoesophageal speech is the most common voicing method used by laryngectomees. This method requires the installation of tracheoesophageal prosthesis (TEP), which requires continuous maintenance to achieve optimal speaking abilities and prevent fluid leakage from the esophagus to the trachea. The present manuscript describes the available types of TEPs, the procedures used to maintain them, the causes for their failure due to fluid leakage, and the methods used for their prevention. Knowledge and understanding of these issues can assist the otolaryngologist in caring for laryngectomees who use tracheoesophageal speech.

\section{Background}

Restoring speech communication using a voice prosthesis was a significant medical advancement for laryngectomees. It enables the laryngectomee to create sound again immediately after its insertion. ${ }^{1}$ A one-way valve (tracheoesophageal prosthesis, TEP) is inserted through a previously-created tracheoesophageal puncture connecting the trachea and esophagus in those wishing to speak through tracheoesophageal speech. When the patient occludes the stoma, the exhaled air is shunted through the TEP into the esophagus, where it induces vibration of the upper esophageal sphincter.

The main advantage of TEP is that it generates the most intelligible, fluent, natural sounding voice in contrast to other alaryngeal speech methods. ${ }^{2}$ Even though esophageal speech also uses the upper esophageal sphincter as the sound source, laryngectomees using a TEP rely on pulmonary air for speech production, rather than the limited air supply available in esophageal speech. This leads to increased fluency and longer utterance lengths. ${ }^{3} \mathrm{~A}$ disadvantage of tracheoesophageal speech is that it requires continuous care and maintenance. The patient has to clean and care for the TEP daily, and the prosthesis needs to be replaced at regular intervals. ${ }^{4}$ Those who rely on the speech and language pathologist (SLP) for TEP changes may have to be seen in the clinic on average every two to three months. ${ }^{5}$ Furthermore, patients with stricture or narrowing of the upper esophageal sphincter region may not be able to achieve good voice.

Tracheoesophageal puncture can be performed at the time of the laryngectomy (primary puncture) or at a later date (secondary puncture). The advantages of placing primary TEP are that individuals are not subjected to an additional surgical procedure, and they can start speech rehabilitation shortly after laryngectomy. However, primary TEP is associated with an increased risk of fistula formation, leakage at the puncture site, stomal stenosis, and local infection. ${ }^{6}$ Additionally, In those who undergo secondary puncture, the SLP has the advantage of determining the tracheoesophageal voice quality before the procedure. ${ }^{6-8}$ This can assist in determining if the tracheoesophageal voice will be acceptable/functional.

Not every laryngectomee is able to use voice prosthesis. ${ }^{4}$ The relative contraindications for voice prosthesis include: received

June 27, 2019

accepted

November 21, 2019
DOI https://doi.org/

10.1055/s-0039-3402497. ISSN 1809-9777.
Copyright $\odot 2020$ by Thieme Revinter

Publicações Ltda, Rio de Janeiro, Brazil
License terms

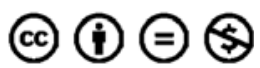


poor dexterity, eye sight, and pulmonary function; impaired mental status; lack of motivation; inability to manage associated care of stoma and voice prosthesis; voicing difficulties; recurrent aspiration and dislodging of the TEP; difficulty in occluding the stoma; proximity of the speech pathologist or otolaryngologist; the lack of support system; and the potential cost and lack of reimbursement.

The assessments needed before the insertion of the TEP include consideration of the patient's surgical history and exposure to radio-chemotherapy, the condition of the upper esophageal sphincter (the presence of pain or dysphagia), and examination of the stoma (size, regularity and signs of infection) and the TEP (location, free rotation, patency and fluid leak). ${ }^{9}$

Tracheoesophageal prostheses last only a limited period of time, and require repeated replacements. They require continuous maintenance by the laryngectomee to achieve optimal speaking abilities and prevent fluid leakage from the esophagus to the trachea because of buildup of biofilm by yeast and bacteria. ${ }^{10}$ Extending the lifespan of the TEP can reduce the medical expenses associated with its replacement.

The present manuscript describes the available types of TEPs, the procedures used to maintain them, the causes for their failure due to fluid leakage from the trachea to the esophagus, and the methods used for their prevention. Knowledge and understanding of these issues can assist the otolaryngologist in caring for laryngectomees who use tracheoesophageal speech.

\section{Types of Voice Prosthesis}

There are two types of voice prosthesis: an indwelling one that is installed and changed by an SLP or otolaryngologist, and a patient-changed one. ${ }^{1,10}$ The indwelling prosthesis generally lasts a longer time than the patient-managed device. An indwelling prosthesis can function well for weeks to months. The patient-managed voice prosthesis enables a greater degree of independence. It can be changed by the laryngectomee on a regular basis (every 1 to 2 weeks). The old prosthesis can be cleaned and reused several times.

\section{Causes of Voice Prosthesis Leak}

There are two patterns of voice prosthesis leak: leak through the prosthesis and leak around it. ${ }^{10}$

Leakage through the voice prosthesis is predominantly due to situations in which the valve can no longer close tightly. This may be due the following: colonization of the valve by fungal-bacterial biofilm; the flap's valve may get stuck in the open position; a piece of food, mucus or hair (in those with a free flap) stuck on the valve; or the device coming in contact with the posterior esophageal wall. Inevitably, all prostheses will fail by leaking through, whether from Candida biofilm colonization or simple mechanical failure. ${ }^{11}$

If there is continuing leakage through the prosthesis from the time it is inserted, the problem is generally caused by the flap's valve remaining open because of the negative pressure generated by swallowing. ${ }^{10}$ This can be corrected by using a prosthesis that has a greater resistance. The trade-off is that having such a voice prosthesis may require more effort when speaking. It is nevertheless important to prevent chronic leakage that can lead to aspiration into the lungs.

Leakage around the voice prosthesis is less common and is mainly due to TEP tract dilation or the inability to grip the prosthesis. $^{12}$ It has been linked to shorter prosthesis life span. It may occur when the puncture that houses the prosthesis widens. During insertion of the voice prosthesis, some dilation of the puncture takes place, but if the tissue is healthy and elastic, it should shrink back after a short time. The inability to contract back can be associated with gastroesophageal reflux, poor nutrition, alcoholism, hypothyroidism, improper puncture placement, incorrectly-fitted prosthesis, TEP tract trauma, local granulation tissue, recurrent or persistent local or distant cancer, past radiation treatment, and radiation necrosis. ${ }^{10}$

Leakage around the prosthesis can also occur if the prosthesis is too long for the user's tract. Whenever this occurs, the voice prosthesis moves back and forth in the tract (pistoning), thereby dilating it. ${ }^{12}$ The tract should be measured, and a prosthesis of more appropriate length should be inserted. In this circumstance, leakage should resolve within 48 hours. If the tissue around the prosthesis does not heal around the shaft within this period, a comprehensive medical evaluation is warranted to determine the cause of the problem.

Another cause of leakage around the prosthesis is the presence of stricture of the esophagus. The narrowing of the esophagus forces the laryngectomee to swallow harder, with greater force, so that the food/liquid goes through the stricture. The excess swallowing pressure pushes the food/liquid around the prosthesis. ${ }^{12}$

Uncontrolled gastroesophageal reflux can limit the voice prosthesis life span. It is advisable to treat gastroesophageal reflux..$^{13}$ Treatment of reflux can allow the esophageal tissue to heal.

Several procedures have been used to treat persistent leakage around the prosthesis. These include temporary removal of the prosthesis and replacement with a smallerdiameter catheter to encourage spontaneous shrinkage; using customized prostheses; placing a purse-string suture around the puncture; injection of gel, collagen or micronized AlloDerm (LifeCell, Branchburg, NJ, US); ${ }^{14}$ cautery with silver nitrate or electrocautery; autologous fat transplantation; inserting a larger prosthesis to stop the leak; and surgical or non-surgical (removing the prosthesis, allowing closure to occur) closure of the puncture. Granulation tissue can be removed by cauterization (electro-, chemo-, laser-).

Increasing the diameter of the prosthesis is generally not recommended. Some, however, believe that using a largerdiameter prosthesis reduces the speaking pressure (the larger diameter enables a better airflow), which allows greater tissue healing to occur while the underlying cause (most often reflux) is treated. ${ }^{15}$ The use of a prosthesis with a larger esophageal and/or tracheal flange may be helpful, as the flange acts as a washer to seal the prosthesis against the 
walls of the esophagus and/or trachea, thus preventing leakage.

Patients with a TEP need to be followed by an SLP because of normal changes in the tracheoesophageal tract. Resizing of the tract may be needed as it can change in length and diameter with time. ${ }^{16}$ The length and diameter of the prosthesis' puncture generally change over time, as the swelling generated by the creation of the fistula, the surgery and the radiation gradually decreases. This requires repeated measurements of the length and diameter of the puncture tract by the SLP, who can select a properly-sized prosthesis.

\section{Cleaning the Voice Prosthesis and Preventing Leakage}

It is very important to keep the voice prosthesis clean to insure its proper function and durability. When not cleaned properly, the prosthesis can leak, and the ability to speak can be compromised or weakened. It is recommended that the voice prosthesis be cleaned at least twice a day (morning and evening), and preferably after eating, because this is the time when food and mucus can become trapped. Cleaning is especially helpful after eating sticky foods or whenever one's voice is weak. ${ }^{17} \mathrm{~A}$ prosthesis cleaning brush and flushing bulb are used in the cleaning process.

It is advisable to clean the voice prosthesis' inner lumen at least twice a day and after each meal. Warm water works better than room temperature water in cleansing the prosthesis, probably because it dissolves the dry secretions and mucus and perhaps even flushes away (or even kills) some of the yeast colonies that had formed on the prosthesis. ${ }^{17}$

Initially the mucus around the prosthesis should be cleaned using tweezers, preferably with rounded tips. Following that, the manufacturer-provided brush should be inserted into the prosthesis and twisted back and forth. ${ }^{18}$ The brush should be thoroughly washed with warm water after each cleaning. The prosthesis is then flushed twice with warm (not hot) water using the manufacturer's provided bulb. The flushing bulb should be introduced into the prosthesis opening while applying slight pressure to completely seal off the opening. The angle in which one should place the tip of the bulb varies between individuals. (The SLP can provide instructions on how to choose the best angle.) Flushing the prosthesis should be performed gently, because using too much pressure can lead to splashing of water into the trachea. If flushing with water is problematic, the flush can also be used with air.

The manufacturers of each voice prosthesis brush and flushing bulb provide directions on how to clean them and when they should be discarded. The brush should be replaced when its threads become bent or worn out. ${ }^{16}$ The prosthesis brush and flushing bulb should be cleaned with hot water, when possible, and soap, and dried with a towel after every use. One way to keep them clean is to place them on a clean towel and expose them to sunlight for a few hours, on a daily basis. This takes advantage of the antibacterial power of the sun's ultraviolet light to reduce the number of bacteria and fungi.
Placing $2 \mathrm{~mL}$ to $3 \mathrm{~mL}$ of sterile saline in the trachea at least twice a day (and more if the air is dry), wearing a heat and moisture exchanger (HME) 24/7 and using a humidifier can keep the mucus moist and reduce the clogging of the voice prosthesis.

\section{Preventing Yeast and Bacteria Biofilm from Growing on the Voice Prosthesis}

Overgrowth of yeast and bacteria in the form of a biofilm on the voice prosthesis is one cause of prosthesis leakage and thus failure. Nevertheless, it takes some time for yeast and bacteria to grow in a newly installed voice prosthesis and form the biofilm that prevent its valve from closing completely. ${ }^{7}$ Accordingly, failures immediately after voice prosthesis installation are unlikely due to yeast growth.

The presence of yeast should be established by the person who changes the failing voice prosthesis. This can be done by observing the typical yeast (Candida) colonies that prevent the valve from closing and, if possible, by sending a specimen from the voice prosthesis for fungal culture. ${ }^{8,9}$ Mycostatin (an antifungal agent) is often used to prevent voice prosthesis failure due to yeast. It is available with a prescription in the form of a suspension or tablets. The tablets can be crushed and dissolved in water. There is anecdotal information that apple cider vinegar, which is known to inhibit Candida growth, can be used to gargle and be swallowed to prevent yeast growth on the TEP.

Automatically administering antifungal therapy just because one assumes that yeast is the cause of voice prosthesis failure may be inappropriate without proof. It is expensive, may lead to the yeast developing resistance to the agent, and may cause unnecessary side effects. ${ }^{8}$ There are, however, exceptions to this rule. These include the administration of preventive antifungal agents to diabetics, those receiving antibiotics, chemotherapy or steroids, and those in whom colonization with yeast is evident (coated tongue etc.).

There are several methods that help prevent yeast from growing on the voice prosthesis: ${ }^{10}$

- Reduce the consumption of sugars in food and drinks, and brush your teeth well after consuming sugary food and/or drinks.

- Brush your teeth well after every meal and especially before going to sleep.

- Clean your dentures daily.

- Diabetics should control blood sugar levels.

- Generally avoid antibiotics and corticosteroids, taking only as needed.

- After using an oral suspension of an antifungal agent, wait for 30 minutes to let it work and then brush your teeth. This is because some of these suspensions contain sugar.

- Dip the voice prosthesis brush in a small amount of mycostatin (nystatin) suspension or vinegar and brush the inner voice prosthesis before going to sleep. (A homemade suspension can be made by dissolving a quarter of a mycostatin tablet in $3 \mathrm{~mL}$ to $5 \mathrm{~mL}$ of water). ${ }^{19}$ This would leave some of the suspension inside the voice prosthesis. 
The unused suspension should be discarded. Do not place too much mycostatin or vinegar in the prosthesis to prevent dripping into the trachea. Speaking a few words after placing the suspension will push it towards the inner part of the voice prosthesis.

- Consume probiotics by eating active-culture yogurt. ${ }^{20}$

- Gently brush the tongue if it is coated with yeast (white plaques).

- Replace the toothbrush after overcoming a yeast problem to prevent recolonization.

- Keep the prosthesis brush clean.

\section{The Use of Probiotics Such as Lactobacillus acidophilus to Prevent Yeast Overgrowth}

A probiotic that is often used to prevent yeast overgrowth is a preparation containing the viable bacteria Lactobacillus acidophilus. However, there is no FDA-approved indication to use $L$. acidophilus to prevent yeast growth. This means that there were no controlled studies to ensure its safety and efficacy. L. acidophilus preparations are sold as a nutritional supplement and not as a medication. The recommended dosage is between 1 and 10 billion bacterial colony-forming units (CFUs). ${ }^{8}$ Typically, L. acidophilus tablets contain a dosage somewhere within this recommended amount of bacteria. Dosage suggestions vary by the tablet's brand, but generally it is advised to take between one and three tablets daily. $^{20}$

Although generally believed to be safe with few side effects, oral preparations of $L$. acidophilus should be avoided in people with intestinal damage, a weakened immune system, or with overgrowth of intestinal bacteria. ${ }^{21}$ In these individuals, this bacterium can cause serious and sometimes life-threatening complications. This is why individuals should consult their physician whenever this live bacteria is ingested. It is especially important in those with the aforementioned conditions.

\section{Conclusions}

Restoring speech communication using a voice prosthesis provides the laryngectomee with the ability to speak using tracheoesophageal voice. The use of TEP requires continuous maintenance, cleaning and replacement in order to maintain voice quality and prevent fluid leakage.

Conflict of Interests

The authors have no conflict of interests to declare.

\section{References}

1 Singer S, Wollbrück D, Dietz A, et al. Speech rehabilitation during the first year after total laryngectomy. Head Neck 2013;35(11): 1583-1590

2 Merwin GE, Goldstein LP, Rothman HB. A comparison of speech using artificial larynx and tracheoesophageal puncture with valve in the same speaker. Laryngoscope 1985;95(06):730-734
3 Sedory SE, Hamlet SL, Connor NP. Comparisons of perceptual and acoustic characteristics of tracheoesophageal and excellent esophageal speech. J Speech Hear Disord 1989;54(02):209-214

4 Saurajen AS, Chee NW, Siow JK, Lim F, Mok PK, Earnest A. Tracheoesophageal puncture outcomes and predictors of success in laryngectomised patients. Ann Acad Med Singapore 2000;29 (04):452-456

5 Lewin JS, Baumgart LM, Barrow MP, Hutcheson KA. Device life of tracheoesophageal voice prosthesis revisited. JAMA Otolaryngol Head Neck Surg 2017;143(01):65-71

6 Barauna Neto JC, Dedivitis RA, Aires FT, Pfann RZ, Matos LL, Cernea CR. Comparison between Primary and Secondary Tracheoesophageal Puncture Prosthesis: A Systematic Review. ORL J Otorhinolaryngol Relat Spec 2017;79(04):222-229

7 Blom ED, Singer MI, Hamaker RC. A prospective study of tracheoesophageal speech. Arch Otolaryngol Head Neck Surg 1986;112 (04):440-447

8 Leonhard M, Schneider-Stickler B. Voice prostheses, microbial colonization and biofilm formation. Adv Exp Med Biol 2015; 830:123-136

9 Sayed SI, Datta S, Deore N, Kazi RA, Jagade MV. Prevention of voice prosthesis biofilms: current scenario and future trends in prolonging prosthesis lifetime. J Indian Med Assoc 2012;110(03): 175-178, 180

10 Brook I. The Laryngectomee Guide. ISBN-13: 9781483926940. CreateSpace Publication, Charleston SC. 2013

11 Wells C, Adcock L. Indwelling Voice Prostheses for Adults Following Laryngectomy: A Review of Clinical Effectiveness, Cost-Effectiveness, and Guidelines. Canadian Agency for Drugs and Technologies in Health; 2017 Dec 13. https://www.cadth.ca/indwelling-voice-prostheses-adults-following-laryngectomyreview-clinical-effectiveness-cost

12 Lorenz KJ. The development and treatment of periprosthetic leakage after prosthetic voice restoration: a literature review and personal experience. Part II: conservative and surgical management. Eur Arch Otorhinolaryngol 2015;272(03):661-672

13 Pattani KM, Morgan M, Nathan CO. Reflux as a cause of tracheoesophageal puncture failure. Laryngoscope 2009;119(01): 121-125

14 Namnoum JD. Expander/implant reconstruction with AlloDerm: recent experience. Plast Reconstr Surg 2009;124(02):387-394

15 Naunheim MR, Remenschneider AK, Scangas GA, Bunting GW, Deschler DG. The Effect of Initial Tracheoesophageal Voice Prosthesis Size on Postoperative Complications and Voice Outcomes. Ann Otol Rhinol Laryngol 2016;125(06):478-484

16 Jiang N, Kearney A, Damrose EJ. Tracheoesophageal fistula length decreases over time. Eur Arch Otorhinolaryngol 2016;273(07): 1819-1824

17 Friedlander E, Pinacho Martínez P, Poletti Serafini D, MartínOviedo C, Martínez Guirado T, Scola Yurrita B. Practical management of periprosthetic leakage in patients rehabilitated with a Provox ${ }^{\circledR} 2$ voice prosthesis after total laryngectomy. Acta Otorrinolaringol Esp 2016;67(06):301-305

18 Balm AJ, van den Brekel MW, Tan IB, Hilgers FJ. The indwelling voice prosthesis for speech rehabilitation after total laryngectomy: a safe approach. Otolaryngol Pol 2011;65(06):402-409

19 Messing BP, Kim M, Hirata R, et al. Evaluation of prophylaxis treatment of Candida in alaryngeal patients with tracheoesophageal voice prostheses. Laryngoscope 2015;125(05):1118-1123

20 Schwandt LQ van Weissenbruch R, van der Mei HC, Busscher HJ, Albers FW. Effect of dairy products on the lifetime of Provox2 voice prostheses in vitro and in vivo. Head Neck 2005;27(06): 471-477

21 Franko B, Fournier P, Jouve T, et al. Lactobacillus bacteremia: Pathogen or prognostic marker? Med Mal Infect 2017;47(01): $18-25$ 